\title{
Es tut sich etwas im europäischen Gesundheitswesen
}

Die Probleme im Gesundheitswesen sind in der EU nicht mehr ausschliesslich ein nationales Thema. Zurzeit laufen Überlegungen in mehreren Stossrichtungen.

Die erste bezieht sich auf die Mobilität von Patienten und Gesundheitsdienstleistungen. Die Europäische Kommission hat zu diesem Thema eine Vernehmlassung bis am 31. Januar 2007 gestartet. Dabei stellt sich natürlich nicht nur die Frage nach den Versicherungsmodalitäten der verschiedenen Länder, abgesehen von Notfallbehandlungen, sondern vor allem das Problem des Datenverkehrs. Die europäische Versicherungskarte stellt hier einen ersten Schritt dar. Zurzeit ersetzt sie nur das E-111-Formular mit den administrativen Angaben. Letztlich geht es jedoch um das gesamte Thema der medizinischen Daten und allgemein um die elektronischen Informationen. Der Ständige Ausschuss der Europäischen Ärzte (CPME) verfolgt diese Entwicklung besonders aufmerksam.

Im Mittelpunkt steht dabei auch die Patientensicherheit. $\mathrm{Zu}$ diesem Thema stiess eine gemeinsame Erklärung, die von den verschiedenen europäischen Gesundheitsorganisationen (Gesundheitsberufe, Konsumenten und CPME) im Juni 2005 in Luxemburg verabschiedet wurde, bei der Generaldirektion Gesundheit und Konsumentenschutz (DG-Sanco) der Europäischen Kommission auf ein positives Echo. Je nach Reaktionen im Rahmen der laufenden Vernehmlassung werden eine oder mehrere Richtlinien zu diesen Fragen ausgearbeitet.

Schliesslich wurde im Juni 2005 im Zuge der EU-Präsidentschaft Grossbritanniens in Edinburgh ein Gentlemen's Agreement über den freien Personenverkehr für die Ärzte geschlossen. Unter dem finnischen Vorsitz wurden am 23. Oktober 2006 die Vertreter der EU-Mitgliedstaaten nach Helsinki eingeladen, um die Umsetzungsmodalitäten festzulegen. Dabei geht es um die beruflichen Qualifikationen gemäss den Regeln der Richtlinie 2005/35/EG über die gegenseitige Anerkennung der Diplome sowie die Situation der Auswanderungswilligen bezüglich der berufsethischen und rechtlichen Aspekte. Es darf selbstverständlich nicht sein, dass einem Kollegen oder einer Kollegin in einem anderen Land als dem Herkunftsland eine Praxisbewilligung erteilt wird, wenn damit Sanktionen des Berufsverbandes oder ein Strafverfahren umgangen werden sollen.

Die bilateralen Verträge sind kein Allheilmittel. Die Schweiz und die FMH sind zwar insbesondere über die CPME, der wir als Vollmitglied angehören, bis zu einem gewissen Grad an den Diskussionen beteiligt. Leider können wir jedoch an den eigentlichen Entscheidungen nicht mitwirken. Diese müssen aber im Rahmen unserer Institutionen übernommen werden, insbesondere von den durch das Bundesgesetz über die universitären Medizinalberufe (MedBG) geschaffenen Instanzen in bezug auf die Anerkennung der ausländischen Diplome und Ausbildungen. Die FMH verfolgt deshalb diese Angelegenheiten mit grösster Aufmerksamkeit, da sie für die Ausübung unseres Berufs sowie die künftige Entwicklung des gesundheitspolitischen Umfelds in der Schweiz von entscheidender Bedeutung sind.

Aus Anlass der ordentlichen Herbstsitzung der CPME, Luxemburg, 28. Oktober 2006.

Dr. med. Yves Guisan Vizepräsident der FMH, Nationalrat 